UM-TH-94-31

August 1994

hep-ph/9408380

\title{
More on ambiguities in the pole mass
}

\author{
M. BENEKE \\ Randall Laboratory of Physics \\ University of Michigan \\ Ann Arbor, Michigan 48109, U.S.A.
}

\begin{abstract}
The relation between the pole quark mass and the $\overline{M S}$-renormalized mass is governed by an infrared renormalon singularity, which leads to an ambiguity of order $\Lambda_{Q C D}$ in the definition of the pole mass. We use the renormalization group and heavy quark effective theory to determine the exact nature of this singularity up to an overall normalization. In the abelian gauge theory, the normalization is computed partially to next-to-leading order in the flavour expansion.
\end{abstract}

submitted to Physics Letters B 
1. It is well known that perturbative series in QCD diverge. Recently there has been a resurge of interest in learning about nonperturbative effects in a variety of situations through this divergence. Among the quantities that have become an object of scrutiny is the pole mass of a quark, defined perturbatively as the location of the singularity of the renormalized quark propagator. The pole mass can be related to the $\overline{\mathrm{MS}}$ renormalized mass - which in principle can be measured to any accuracy at a very high energy scale - by the series']

$$
m_{\mathrm{pole}}=m_{\overline{\mathrm{MS}}}+\sum_{n=0}^{\infty} r_{n} \alpha^{n+1}
$$

where the first two coefficients $r_{0}$ and $r_{1}$ are known [1]. Being infrared (IR) finite and scheme-independent [2], the pole mass is the natural mass parameter in heavy quark systems, whose scale is governed by the mass of the heavy quark. Moreover, the pole mass is implicit in the construction of heavy quark effective theory (HQET), which has become the major theoretical framework for the description of heavy hadron decays [3].

Recently, investigation of the set of diagrams symbolized by Fig. 1a revealed that in large orders, the coefficients $r_{n}$ are strongly contributed by IR momenta in the integration over the gluon line and diverge as [4, 5]

$$
r_{n} \stackrel{n \rightarrow \infty}{=} \frac{C_{F}}{\pi} e^{5 / 6} \mu\left(-2 \beta_{0}\right)^{n} n !,
$$

where $\beta_{0}$ is the first coefficient of the $\beta$-function [We use $\beta(\alpha) \equiv \mu^{2}(\partial \alpha) /\left(\partial \mu^{2}\right)=$ $\beta_{0} \alpha^{2}\left(1+\sum_{n=1} c_{n} \alpha^{n}\right) . C_{F}=4 / 3$ for SU(3) and $C_{F}=1$ for U(1).]. If one attempts to sum the divergent series with the help of the Borel transform, the Borel sum

$$
m_{\text {pole }}=m_{\overline{\mathrm{MS}}}+\int_{0}^{\infty} \mathrm{d} t e^{-t / \alpha} B\left[m_{\text {pole }}\right](t) \quad B\left[m_{\text {pole }}\right](t) \equiv \sum_{n=0}^{\infty} r_{n} \frac{t^{n}}{n !},
$$

is ambiguous of order $\Lambda_{\mathrm{QCD}}$ from a simple IR renormalon pole at $t=-1 /\left(2 \beta_{0}\right)$. In the following we adopt the convention that all quantities that have divergent expansions are understood as Borel sums as in Eq. (3) with the contour deformed above the singularities on the positive axis. Then the ambiguity of order $\Lambda_{\mathrm{QCD}}$ is equivalently expressed as an imaginary part of $m_{\text {pole }}$ of this order.

The ambiguity in the definition of the pole mass can immediately be translated into an ambiguity of the parameter $\bar{\Lambda}_{H_{Q}}$, which appears in HQET, if defined as usual through the expansion of the heavy hadron mass

$$
m_{H_{Q}}=m_{\text {pole }}+\bar{\Lambda}_{H_{Q}}+O\left(1 / m_{\text {pole }}\right) .
$$

Physically, this ambiguity of $\bar{\Lambda}_{H_{Q}}$ arises, because the contribution of the spectator to the mass of the hadron can not be separated from self-energy corrections to the heavy quark, once these become sensitive to the long range part of the Coulomb potential.

\footnotetext{
${ }^{1} \mathrm{~A}$ normalization point $\mu$ is understood for $m_{\overline{\mathrm{MS}}}$ and the $\overline{\mathrm{MS}}$ coupling $\alpha$.
} 
This fact is known in a different language to the lattice community, where the extraction of the constant term in the heavy quark potential has faced difficulties precisely because of the contamination from self-energy contributions.

In this letter, we address a more formal issue and explore to what extent the large order behaviour of the coefficients $r_{n}$ can be determined without any approximation. In general, one expects the behaviour of Eq. (2) to be modified to

$$
r_{n} \stackrel{n \rightarrow \infty}{=} N \mu\left(-2 \beta_{0}\right)^{n} \Gamma(n+1+b)\left(1+\sum_{k=1}^{\infty} \frac{s_{k}}{n^{k}}\right),
$$

turning the simple pole of $B\left[m_{\text {pole }}\right](t)$ at $t=-1 /\left(2 \beta_{0}\right)$ into a branch point of strength $1+b$. We shall determine the exact nature of the singularity, i.e. $b$ and the $s_{k}$, entirely in terms of the $\beta$-function. The analoguous coefficient $b$ is known to date only for the QCD corrections to $e^{+} e^{-}$annihilation and the GLS sum rule [6, 7], where, however, the corresponding singularity is not responsible for the dominant divergent behaviour of the series. In these cases one may exploit the short distance expansion of the appropriate current product and a relation to higher dimension operators. The short distance expansion is not available for the pole mass. Instead we will utilize the observation of Ref. [4], that the IR renormalon at $t=-1 /\left(2 \beta_{0}\right)$ in the pole mass is closely related to an ultraviolet (UV) renormalon singularity at the same position in the self-energy of a static quark. The normalization $N$ remains elusive. We calculate it partially to next-to-leading order in the flavour expansion in the abelian theory.

Though we doubt that our results can be used phenomenologically to improve the perturbative relation Eq. (11), we believe that the arguments that lead to the characterization of Eq. (5) are interesting by themselves and may shed more light on the simplicities and complexities of divergent series in renormalizable theories.

2. In this section we consider the self-energy of a static quark, described by the leading order effective Lagrangian of HQET. Its Lorentz covariant form is

$$
\mathcal{L}_{\text {eff }}=\bar{h}_{v} i v \cdot D h_{v}+\mathcal{L}_{\text {light }},
$$

where $v$ is the quark velocity and $\mathcal{L}_{\text {light }}$ the QCD Lagrangian for the light degrees of freedom. In contrast to the self-energy of a quark of finite mass, the self-energy of a static quark is linearly UV divergent. Starting from a bare Lagrangian of the above form, renormalization will in general involve a counterterm $\bar{h}_{v} h_{v}$, such that the renormalized Lagrangian contains a residual mass term $\delta m_{\text {res }} \propto \mu$ for the static quark. We choose a minimal subtraction (MS) like subtraction scheme (i.e. $\overline{\mathrm{MS}}$ or schemes that differ from $\overline{\mathrm{MS}}$ by a change of scale $\mu$ ). Then a residual mass does not arise to all orders in perturbation theory, but the subtractions are such that the series expansion of $\Sigma_{\text {eff }}$ has an UV renormalon divergence analogous to Eq. (5) [4]. We emphasize the choice of MS-like schemes. Large order coefficients are scheme-dependent and in general (massdependent) schemes, this particular UV renormalon divergence can be eliminated at the price of introducing a residual mass, which is adjusted precisely to this divergence. 
At this point we return to the old observation by Parisi [8] that the imaginary parts of the Borel-summed Green functions due to an UV renormalon at position $t=n / \beta_{0}$ in the Borel plane are proportional to a sum over zero-momentum insertions of all possible local operators of dimension $2 n+4$, which can be considered as a simple version of a factorization theorem. Generically

$$
\operatorname{Im} G\left(p_{k}\right)=\sum_{d} \sum_{\operatorname{dim} \mathcal{O}_{i}=d} E_{d i} G_{\mathcal{O}_{i}}\left(p_{k}\right)
$$

Although we are not aware of a general proof of this statement, we note that it has been confirmed in a nontrivial case of the photon vacuum polarization [9, 10] as far as dimension six operators are concerned. In fact, Eq. (7) is almost intuitive, if one understands the emergence of UV renormalons in the context of renormalization in general. After the theory is regulated with a dimensionful cutoff, renormalization can be expressed as hiding all divergent terms in a large-cutoff expansion into a redefinition of the low-energy parameters. The remaining cutoff-dependence determines the remaining sensitivity to small distances, which is suppressed by a power of the cutoff. It is these "left-overs", which give rise to divergent series, when the series is expressed in terms of the renormalized low-energy coupling. However, in principle, order by order in the inverse cutoff the remaining sensitivity to small distances could be removed by adding local higher dimension operators to the Lagrangian, a procedure that is indeed widely used in the construction of improved lattice actions. By the same token, the imaginary parts created by UV renormalons can be described by Eq. (7).

In the particular case of the self-energy, the situation is in fact much simpler. The UV renormalon at $t=-1 /\left(2 \beta_{0}\right)$ is related to the dominant (linear) cutoff-dependent term and one does not have to deal with a small left-over from renormalization. As indicated by the position of the pole and the discussion above, the relevant operators have dimension three. There exists only one dimension three operator, $\bar{h}_{v} h_{v}$, and the general expression Eq. (7) reduces to

$$
\operatorname{Im} \Sigma_{\mathrm{eff}}(v k, \mu, \alpha)=E(\mu, \alpha) \Sigma_{\mathrm{eff}, \bar{h}_{v} h_{v}}\left(\frac{v k}{\mu}, \alpha\right),
$$

as far as the renormalon at $t=-1 /\left(2 \beta_{0}\right)$ alone is concerned. Here $\Sigma_{\text {eff }, \bar{h}_{v} h_{v}}$ denotes the self-energy with one zero-momentum insertion of $\bar{h}_{v} h_{v}$. Eq. (8) is illustrated to leading order in Fig. 2. Comparing the renormalization group equations for $\Sigma_{\text {eff }}$ (and therefore its imaginary part) and $\Sigma_{\text {eff }, \bar{h}_{v} h_{v}}$, one finds that the coefficient satisfies

$$
\left(\mu^{2} \frac{\partial}{\partial \mu^{2}}+\beta(\alpha) \frac{\partial}{\partial \alpha}-\gamma_{\bar{h}_{v} h_{v}}(\alpha)\right) E(\mu, \alpha)=0 .
$$

The anomalous dimension $\gamma_{\bar{h}_{v} h_{v}}(\alpha)$ of the operator $\bar{h}_{v} h_{v}$ vanishes to all orders in perturbation theory, because the operator is related to the conserved current $\bar{h}_{v} \gamma_{\mu} h_{v}$ by spin symmetry. A different way to see this is to observe that $m_{Q} \bar{h}_{v} h_{v}$ is renormalization group invariant, but the HQET expansion parameter $m_{Q}$ does not run. By dimensional arguments, the explicit $\mu$-dependence of $E$ must be an overall factor $\mu$ and the solution of Eq. (9) is 


$$
E(\mu, \alpha)=\text { const } \times \mu \exp \left(\int_{\alpha} \mathrm{d} \alpha^{\prime} \frac{1}{2 \beta\left(\alpha^{\prime}\right)}\right)=\text { const } \times \Lambda_{\mathrm{QCD}} .
$$

Together with Eq. (8) this determines completely the UV renormalon singularity of the self-energy up to an overall constant in terms of the $\beta$-function and the perturbative expansion of $\Sigma_{\text {eff, } \bar{h}_{v} h_{v}}$. Note that it depends on $v k / \mu$ only through $1 / n$-corrections to the leading asymptotic behaviour in accordance with the expectation that the divergence of the derivative of the self-energy is suppressed, because it is linearly divergent only through linearly divergent subdiagrams. For later use, we note the equality

$$
\Sigma_{\mathrm{eff}, \bar{h}_{v} h_{v}}=1-\frac{\partial \Sigma_{\mathrm{eff}}}{\partial v k}
$$

which can easily be shown diagrammatically by routing the external momentum $k$ through the heavy quark line. The unity on the r.h.s. appears, because the tree diagram on the r.h.s. of Fig. 2 is included in the definition of $\Sigma_{\text {eff, } \bar{h}_{v} h_{v}}$.

3. We now relate the UV renormalon in the self-energy of a static quark discussed above to the leading IR renormalon in the pole mass, generalizing the leading order observation of Ref. [由]. To this end we sandwich the full inverse propagator $S^{-1}$ of a quark in QCD between two projectors $P_{v}=(1+\not) / 2$ and define

$$
P_{v} S^{-1}\left(p, m_{\overline{\mathrm{MS}}}, \mu ; \alpha\right) P_{v} \equiv P_{v} S_{\mathrm{P}}^{-1}\left(v k, m_{Q}, \mu ; \alpha\right)
$$

with $p=m_{Q} v+k$. As long as $p$ is real and away from the zero of $S^{-1}$, (the Borel sum of) $S^{-1}$ does not have an imaginary part of order $\Lambda_{\mathrm{QCD}}$, because its Borel transform has no singularity at $t=-1 /\left(2 \beta_{0}\right)$. Odd powers of $\Lambda_{\mathrm{QCD}}$ can arise only from on-shell Feynman integrals or expansion around mass-shell. For the purpose of our argument, we shall assume the hierarchy $m_{Q} \gg v k \gg \Lambda_{\mathrm{QCD}}$. Then the largest imaginary part of $S_{\mathrm{P}}^{-1}$ comes from a singularity of its Borel transform at $t=-1 / \beta_{0}$, which is or order $\Lambda_{\mathrm{QCD}}^{2} / v k$, parametrically smaller than $\Lambda_{\mathrm{QCD}}$.

We wish to expand $S_{\mathrm{P}}^{-1}$ in $v k / m_{Q}$ about $v k=0$, where $p, m_{Q}$ and $v k$ should be real. Anticipating an imaginary part of the Borel sum of $m_{\text {pole }}$ of order $\Lambda_{\mathrm{QCD}}$, we define

$$
m_{Q} \equiv m_{\text {pole }}-\delta \hat{m} \quad \delta \hat{m} \equiv i \delta m \equiv i \operatorname{Im} m_{\text {pole }}
$$

To all orders in perturbation theory, $S_{\mathrm{P}}^{-1}$ can be matched onto the self-energy of a static quark computed from HQET without a residual mass term, Eq. (6), and expansion parameter $m_{Q}=m_{\text {pole }}$. Thus defining $k^{\prime}=k-\delta \hat{m} v$ and using $\delta m \ll v k$, one obtains

$$
\begin{aligned}
S_{\mathrm{P}}^{-1}\left(v k, m_{Q}, \mu\right) & =C\left(\frac{m_{\text {pole }}}{\mu}\right)\left(v k^{\prime}-\Sigma_{\text {eff }}\left(v k^{\prime}, \mu\right)\right)+O\left(\frac{\left|k^{\prime}\right|^{2}}{m_{\text {pole }}}\right) \\
& =C\left(\frac{m_{Q}}{\mu}\right)\left[v k-\Sigma_{\text {eff }}(v k, \mu)-\delta \hat{m}\left(1-\frac{\partial \Sigma_{\text {eff }}}{\partial v k}(v k / \mu)\right)\right]
\end{aligned}
$$




$$
+\frac{\delta \hat{m}}{\mu} C^{\prime}\left(\frac{m_{Q}}{\mu}\right)\left[v k-\Sigma_{\text {eff }}(v k, \mu)\right]+O\left(\frac{(\delta m)^{2}}{m_{Q}}, \frac{|k|^{2}}{m_{Q}}\right),
$$

with a matching coefficient $C$ that contains all $m_{Q}$-dependence. While $\Sigma_{\text {eff }}$ contains an imaginary part of order $\Lambda_{\mathrm{QCD}}$, the l.h.s. of Eq. (14) does not. Investigating the scaling of all potential imaginary parts in quantities on the r.h.s. of Eq. (14) with $v k, \mu$ and $\Lambda_{\mathrm{QCD}}$, one finds that the condition that imaginary parts of order $\Lambda_{\mathrm{QCD}}$ cancel, can be expressed as

$$
\operatorname{Im}\left[v k-\Sigma_{\text {eff }}(v k, \mu)-\delta \hat{m}\left(1-\frac{\partial \Sigma_{\text {eff }}}{\partial v k}(v k / \mu)\right)\right]=0
$$

Combining this condition with Eqs. (8) and (11), we obtain the final result

$$
\begin{aligned}
\operatorname{Im} m_{\text {pole }} & =\delta m=-E(\mu, \alpha)=\text { const } \times \Lambda_{\mathrm{QCD}} \\
& =\text { const } \times \mu e^{1 /\left(2 \beta_{0} \alpha\right)} \alpha^{c_{1} /\left(2 \beta_{0}\right)}\left(1+\frac{c_{2}-c_{1}^{2}}{2 \beta_{0}} \alpha+\ldots\right),
\end{aligned}
$$

where $E$ is defined by Eq. (8) and given by Eq. (10). The second line of Eq. (16) is easily translated into the form of the singularity of $B\left[m_{\text {pole }}\right](t)$ at $t=-1 /\left(2 \beta_{0}\right)$, and therefore into the large order behaviour of the coefficients $r_{n}$, Eq. (5), which relates the pole mass to the $\overline{\mathrm{MS}}$ mass 2 . From Eq. (10) or (16) one deduces that except for the overall normalization $N$ all quantities that characterize Eq. (5) are determined by the $\beta$-function. For instance,

$$
b=-\frac{c_{1}}{2 \beta_{0}} \quad s_{1}=\frac{c_{1}^{2}-c_{2}}{4 \beta_{0}^{2}}
$$

and, in general, $1 / n^{k}$-corrections to the leading asymptotic behaviour involve the $\beta$ function coefficients up to $c_{k+1}$. We stress that these conclusions follow with almost no dynamical input. We used essentially only the linear UV divergence of the self-energy of the static quark. The only "nonperturbative" parameter that remains and is not fixed by very general arguments, is the overall normalization [11, 12]. This is because a nonperturbative approach comparable to the semiclassical expansion for instantoninduced (combinatorically) divergent series is lacking for renormalons.

For the ambiguity present from the perturbative definition of the pole mass, Eq. (16) shows that it is proportional to $\Lambda_{\mathrm{QCD}}$ with no multiplying logarithm and series in $\ln \left(\mu / \Lambda_{\mathrm{QCD}}\right)$, which is in fact the only possible form compatible with scheme-invariance of the pole mass. In our formal derivation, the absence of logarithms is due to the vanishing anomalous dimension of $\bar{h}_{v} h_{v}$, which indeed is directly related to the renormalization group invariance of the pole mass as noted above. The bonus of the formal derivation

\footnotetext{
${ }^{2}$ For this argument, we assume that no "unknown" singularities are present. Then the IR renormalon at $t=-1 /\left(2 \beta_{0}\right)$ lies closest to the origin of the Borel plane and determines the asymptotic behaviour of the perturbative coefficients.
} 
is Eq. (8), which allows to calculate (approximately) the overall normalization from the self-energy in HQET.

As mentioned before, the renormalon ambiguity in $m_{\text {pole }}$ translates into an ambiguity in the $\bar{\Lambda}$-parameter of HQET, see Eq. (田). It is tempting to estimate this ambiguity from a leading order calculation of the normalization. The arbitrariness of this procedure is reflected in the different values, spanning $\delta m_{\text {pole }}=\delta \bar{\Lambda}_{H_{Q}}=(50-300) \mathrm{MeV}$, quoted in Refs. [1, 5] and the present considerations can not improve the expectation that this ambiguity is of order $\Lambda_{\mathrm{QCD}}$.

4. In a nonabelian theory, a systematic approach to calculate the normalization of renormalons is not known. Explicit calculations can be performed in an abelian toy model (QED with $N_{f}$ massless fermion for $\mathcal{L}_{\text {light }}$ ) in an expansion in the number of flavours 5 . To organize this expansion, one defines $a=N_{f} \alpha$ and expands in $a$ and $1 / N_{f}$. The $\beta$-function for the rescaled coupling is $\beta(a)=b_{0} a^{2}\left(1+\hat{c}_{1} a+\ldots\right), b_{0}=1 /(3 \pi)$ and $\hat{c}_{1}=3 /(4 \pi)$. We also expand the normalization

$$
N=N_{0}\left(1+\frac{N_{1}}{N_{f}}+\ldots\right) \quad N_{0}=\frac{C_{F}}{\pi N_{f}} e^{5 / 6},
$$

where $N_{0}$ is minus the asymptotic behaviour of coefficients obtained from the set of self-energy diagrams in Fig. 1a. To next-to-leading order in $1 / N_{f}$, one has to extract the asymptotic behaviour from the diagrams symbolized by Fig. 1b-d, which according to Eqs. (5) and (17) is of the form

$$
r_{n} \stackrel{n \rightarrow \infty}{=} N \mu\left(-2 \beta_{0}\right)^{n}\left[1+\frac{1}{N_{f}}\left\{-\frac{\hat{c}_{1}}{2 \beta_{0}} \ln n+N_{1}\right\}+O\left(\frac{1}{N_{f}^{2}}\right)\right] \times\left(1+O\left(\frac{1}{n}\right)\right) .
$$

A simplification arises, because diagrams $1 \mathrm{c}$ and $1 \mathrm{~d}$ do not contribute to the overall normalization, when only one of the two gauge boson momenta is large. For example, diagram 1c amounts to a radiative correction of the r.h.s. of Fig. 2 and therefore affects the asymptotic behaviour of $\Sigma_{e f f}$ only at the level of $1 / n$-corrections, whereas diagram $1 d$ does not contribute to the UV renormalon at $t=-1 /\left(2 \beta_{0}\right)$ at all, because it has no linearly divergent subdiagrams. The present case is markedly distinct from - and simpler than - the vacuum polarization in QED and its first UV renormalon at $t=1 / \beta_{0}$, where diagrams with two chains of fermion bubbles and one large internal momentum are not suppressed [9]. The reason is that for the self-energy, we are interested in the dominant large-momentum contribution to the Feynman integrals. A contribution to $N_{1}$ from diagrams 1c and d can only come from the region, where both gauge boson momenta are large and of the same order.

Diagrams $1 \mathrm{a}$ and $\mathrm{b}$ are given by the first two terms in the $1 / N_{f}$-expansion of diagram 1a with integration over the full gauge boson propagator. The Borel transform (defined analogous to Eq. (3)) of the class of diagrams obtained by insertion of the full propagator is

${ }^{3}$ The same expansion can also be applied to the nonabelian theory, but can not be taken seriously numerically, since it is singular at $N_{f}=33 / 2$, when $\beta_{0}$ is zero. 


$$
B\left[\Sigma_{\text {eff }}^{\mathrm{a}+\mathrm{b}}\right](t)=-\frac{4 \pi C_{F}}{i N_{f}} \int \frac{\mathrm{d}^{4} q}{(2 \pi)^{4}} \frac{(v k)^{2}-q^{2}}{v(k+q) q^{4}} B\left[\frac{\alpha}{1+\Pi\left(-q^{2} / \mu^{2}, \alpha\right)}\right](t),
$$

as discussed in a general context in Ref. [11]. Expanding the gauge vacuum polarization $\Pi$,

$$
\begin{gathered}
\Pi\left(q^{2}\right)=\Pi_{0}\left(q^{2}\right)+\frac{1}{N_{f}} \Pi_{1}\left(q^{2}\right)+\ldots \\
\Pi_{0}\left(q^{2}\right)=-b_{0} \alpha \ln X \quad X \equiv-\frac{q^{2}}{\mu^{2}} e^{-5 / 3},
\end{gathered}
$$

one finds

$$
B\left[\frac{\alpha}{1+\Pi\left(q^{2}\right)}\right](t)=X^{b_{0} t}-\frac{1}{N_{f}} \int_{0}^{t} \mathrm{~d} s s X^{b_{0} s} B\left[\frac{\Pi_{1}}{\alpha}\right](t-s)+O\left(\frac{1}{N_{f}^{2}}\right),
$$

where the first term corresponds to diagram 1a and the second to $1 \mathrm{~b}$. The Borel transform of $\Pi_{1} / \alpha$ is known [13] and can be written as

$$
B\left[\frac{\Pi_{1}}{\alpha}\right](t)=X^{b_{0} t} F(t)-G(t)
$$

where $F(t)$ is a scheme-independent function given by [13, 14, [4]

$$
F(t)=\frac{8}{3 \pi^{2}} \frac{1}{1-\left(1+b_{0} t\right)^{2}} \sum_{k=2}^{\infty} \frac{(-1)^{k} k}{\left(k^{2}-\left(1+b_{0} t\right)^{2}\right)^{2}} \stackrel{t \rightarrow 0}{=}-\frac{\hat{c}_{1}}{t}
$$

The divergence of $F(t)$ at $t=0$ is regulated by $G(t)$, which is scheme-dependent and without poles in $t$. But, since $\alpha /\left(1+\Pi\left(q^{2}\right)\right)$ is scheme-independent in QED, the scheme-dependence of its Borel transform is compensated by the scheme-dependence of the coupling in the exponent of the inverse Borel transform. Consequently, the subtraction dependence of $G(t)$ can be expressed in terms of the $\beta$-function. Define $F_{\text {reg }}(t) \equiv F(t)+\hat{c}_{1} / t, G_{\text {reg }}(t) \equiv G(t)+\hat{c}_{1} / t$, such that both $F_{\text {reg }}(t)$ and $G_{\text {reg }}(t)$ are finite at $t=0$ and

$$
\begin{aligned}
I(u) & \equiv-\frac{4 \pi C_{F}}{i N_{f}} \int \frac{\mathrm{d}^{4} q}{(2 \pi)^{4}} \frac{(v k)^{2}-q^{2}}{v(k+q) q^{4}} X^{-u} \\
& =\frac{4 \pi C_{F}}{N_{f}} v k\left(-\frac{2 v k}{\mu}\right)^{-2 u} e^{5 u / 3}(-6) \frac{\Gamma(-1+2 u) \Gamma(1-u)}{\Gamma(2+u)} .
\end{aligned}
$$

Then 


$$
\begin{aligned}
B\left[\Sigma_{\text {eff }}^{\mathrm{a}+\mathrm{b}}\right](t)= & I\left(-b_{0} t\right)\left[1-\frac{1}{N_{f}} \int_{0}^{t} \mathrm{~d} s s F_{\mathrm{reg}}(t-s)\right]+\frac{1}{N_{f}} \int_{0}^{t} \mathrm{~d} s s I\left(-b_{0} s\right) G_{\mathrm{reg}}(t-s) \\
& +\frac{\hat{c}_{1}}{N_{f}} \int_{0}^{t} \mathrm{~d} s \frac{s}{t-s}\left(I\left(-b_{0} t\right)-I\left(-b_{0} s\right)\right) .
\end{aligned}
$$

It is simple to extract the leading singular behaviour as $t \rightarrow-1 /\left(2 b_{0}\right)$ from this equation. The term involving $G_{\text {reg }}$ produces a $\ln \left(1+2 b_{0} t\right)$ singularity and contributes only to $1 / n$ corrections in Eq. (19), consistent with the scheme-independence of $N_{1}$. The result is

$$
B\left[\Sigma_{\mathrm{eff}}^{\mathrm{a}+\mathrm{b}}\right](t) \stackrel{t \rightarrow-1 /\left(2 b_{0}\right)}{=}-\frac{C_{F}}{\pi N_{f}} e^{5 / 6} \frac{\mu}{1+2 b_{0} t}\left[1+\frac{1}{N_{f}}\left\{\frac{\hat{c}_{1}}{2 b_{0}} \ln \left(1+2 b_{0} t\right)+A\right\}+\ldots\right]
$$

with

$$
\begin{aligned}
A \equiv & \frac{\hat{c}_{1}}{2 b_{0}}-\int_{0}^{-1 /\left(2 b_{0}\right)} \mathrm{d} s s F_{\mathrm{reg}}\left(-1 /\left(2 b_{0}\right)-s\right)=\frac{27}{8} \ln \frac{4}{3} \\
& +12 \sum_{k=2}^{\infty} \frac{(-1)^{k} k}{\left(k^{2}-1\right)^{2}}\left[\frac{1}{2 k^{2}}-\ln \frac{k^{2}-1}{k^{2}-1 / 4}+\frac{1-3 k^{2}}{2 k^{3}}\left(\operatorname{artanh} k^{-1}-\operatorname{artanh}(2 k)^{-1}\right)\right]
\end{aligned}
$$

The last step consists in converting the singularity of the Borel transform in Eq. (28) into the asymptotic behaviour of the coefficients $r_{n}$. We reproduce correctly the coefficient of the $\ln n$-term in Eq. (19), which can already be deduced from Eq. (16) without any calculation, and find ( $\gamma_{E}$ is Euler-Mascheroni's constant)

$$
N_{1}^{\mathrm{b}}=A-\frac{\hat{c}_{1}}{2 b_{0}} \gamma_{E}=0.6831 \ldots
$$

This concludes the partial evaluation of the normalization in next-to-leading order in the abelian theory. Note that to obtain $N_{1}$ one needs the exact vacuum polarization $\Pi_{1}$, in accordance with general expectations [11]. We repeat that a potential contribution from diagram $\mathrm{c}$ and $\mathrm{d}$ from the region where both internal momenta are large is still missing. The presented calculation of $N_{1}$ should be considered as an illustration how the most complicated diagram b can be easily evaluated, using known results on the vacuum polarization. The Borel transform of diagrams $\mathrm{c}$ and $\mathrm{d}$ is given by $\left(u=-b_{0} t\right.$, $\left.v=-b_{0} s\right)$

$$
\begin{aligned}
B\left[\Sigma_{\text {eff }}^{\mathrm{c}+\mathrm{d}}\right](t)= & \left(\frac{3}{2 \pi N_{f}}\right)^{2} e^{-5 u / 3} v k\left(-\frac{2 v k}{\mu}\right)^{-2 u} \int_{0}^{t} \mathrm{~d} s \frac{\Gamma(-1+2 v) \Gamma(1-v) \Gamma(1-u+v)}{\Gamma(2+v) \Gamma(2+u-v)} \\
& \times\left[\frac{\Gamma(-1+2 u)}{\Gamma(1+2 v)}+2 \frac{\Gamma(-2+2 u)}{\Gamma(2 v)}-\Gamma(-1+2 u-2 v)\right] .
\end{aligned}
$$


However, before one can use this expression, one has to extend the renormalization of the Borel transform [4] to diagrams with two chains.

5. The pole mass or, respectively, the self-energy of a static quark is probably the simplest quantity, for which the leading renormalon can be determined up to a global normalization. Its simplicity might also open a path to tackle the outstanding problem to identify which diagrams in addition to fermion bubbles are responsible for converting the abelian $\beta_{0}$ to its nonabelian value, which is expected on general grounds in Eq. (5). To some extent, this issue can be addressed at order $1 / N_{f}^{2}$ in the flavour expansion of the nonabelian theory. The obvious replacement of one fermion bubble in the diagram la by a gluon or ghost bubble is not sufficient to produce the $1 / N_{f}^{2}$-contribution from expanding $\beta_{0}^{n}$. It would be interesting to check that the two-loop diagram involving the three-gluon vertex dressed by fermion bubbles can make up for the missing piece.

Acknowledgements. I wish to thank V. M. Braun for discussions and acknowledge support by the Alexander-von-Humboldt Foundation. 


\section{References}

[1] N. Gray et. al, Z. Phys. C48 (1990) 673

[2] R. Tarrach, Nucl. Phys. B183 (1981) 384

[3] For reviews, see B. Grinstein, Annu. Rev. Nucl. Part. Sci. 42 (1992) 101; M. Neubert, SLAC-PUB-6263, to appear in Phys. Rep.

[4] M. Beneke and V. M. Braun, Max-Planck-Institut preprint MPI-PhT/94-9 hepph/9402364, to appear in Nucl. Phys. B; M. Beneke, V. M. Braun and V. I. Zakharov, Max-Planck-Institut preprint MPI-PhT/94-18 hep-ph/9405304

[5] I. I. Bigi et al., Phys. Rev. D50 (1994) 2234

[6] A. H. Mueller, Nucl.Phys. B250 (1985) 327; Phys. Lett. B308 (1993) 355

[7] V. I. Zakharov, Nucl. Phys. B385 (1992) 385

[8] G. Parisi, Phys. Lett. B76 (1978) 65; M. C. Bergère and F. David, Phys. Lett. B135 (1984) 412

[9] A. I. Vainshtein and V. I. Zakharov, Univ. of Minnesota preprint TPI-MINN-94/9-T hep-ph/9404248

[10] G. Di Cecio and G. Paffuti, Univ. of Pisa preprint IFUP-TH 31/94 hep-th/9406006

[11] G. Grunberg, Phys. Lett. B304 (1993) 183

[12] M. Beneke, Phys. Lett. B307 (1993) 154

[13] M. Beneke, Nucl. Phys. B405 (1993) 424

[14] D. Broadhurst, Z. Phys. C58 (1993) 339 
This figure "fig1-1.png" is available in "png" format from: http://arxiv.org/ps/hep-ph/9408380v1 

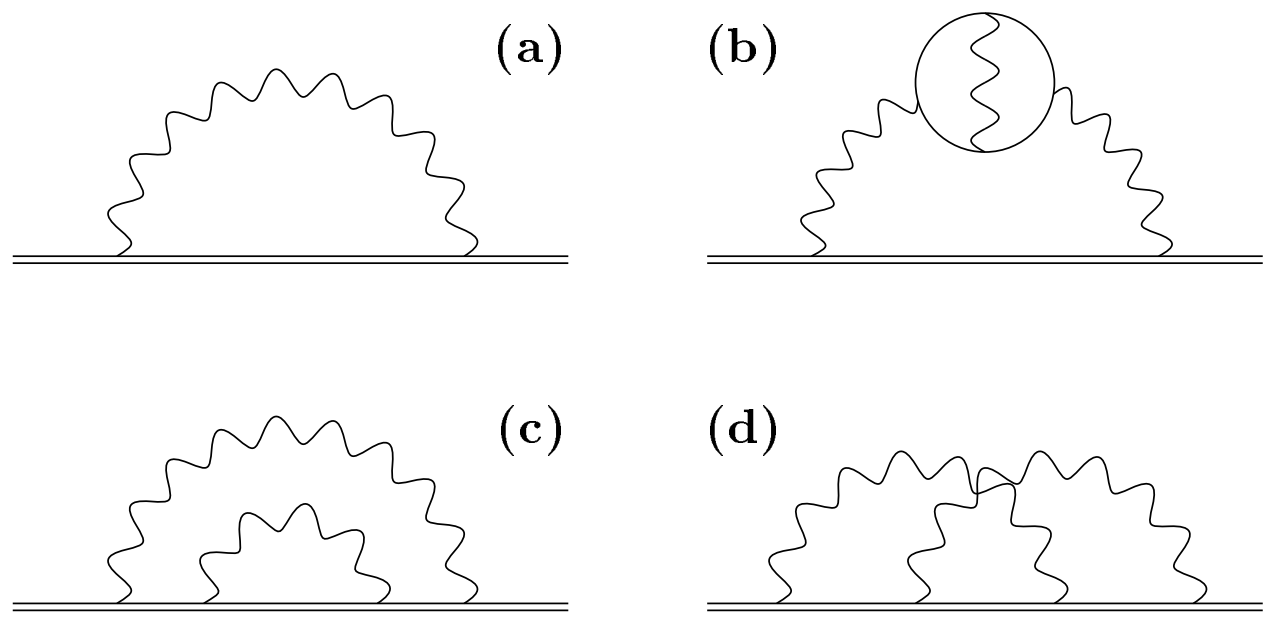

Fig.1 (a) Leading order diagram. (b) - (d) Diagrams to next-to-leading order in the flavour expansion in the abelian theory. Each diagram (a) - (d) represents the set of diagrams obtained by inserting any number of fermion loops in any of the photon (gluon) lines.

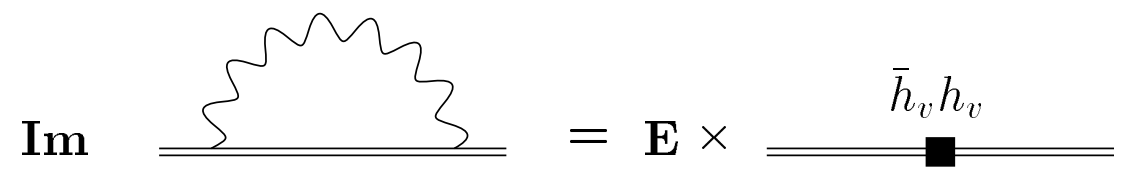

Fig.2 Eq. (8) to lowest order. The gluon line is dressed by a chain of bubbles. 